\title{
Polymer Membranes for Gas Separation: Current Investigations and Future Developments
}

\author{
Denis Rodrigue ${ }^{1,2 *}$ \\ ${ }^{1}$ College of Chemistry and Material Engineering, Hunan University of Arts and Science, China \\ ${ }^{2}$ Department of chemical engineering, Laval University, Canada \\ *Corresponding author: Denis Rodrigue, College of Chemistry and Material Engineering, Hunan University of Arts and Science, China
}

Submission: 眥 February 05, 2019; Published: 眥 February 07, 2019

\begin{abstract}
Although polymer membranes have been used for several decades, they are still under intensive development because of some current limitations. In this review, different applications for gas separation are presented with their main development. The main trends for future investigations are also discussed to conclude.
\end{abstract}

\section{Introduction}

Based on the concept of sustainable development people around the world are more and more concerned about environmental issues. This is especially the case for air and water quality. In particular, the former is of interest for any living being. To this end, several processes have been developed for air purification/gas separation such as cryogenic distillation, adsorption, absorption and membrane technology. The latter is getting more and more interest because of its high efficiency, low power consumption, easy control, simple maintenance and low investment cost compared to the other conventional separation processes. Today, membrane technology can be applied in several industrial fields requiring gas separation/purification such as: oxygen enrichment from air $\left(\mathrm{O}_{2} /\right.$ $\mathrm{N}_{2}$ ), hydrogen separation from a synthesis gas $\left(\mathrm{H}_{2} / \mathrm{CH}_{4}\right.$ or $\left.\mathrm{H}_{2} / \mathrm{CO}_{2}\right)$, hydrogen recovery in ammonia plants $\left(\mathrm{H}_{2} / \mathrm{N}_{2}\right.$ or $\left.\mathrm{H}_{2} / \mathrm{CH}_{4}\right)$, methane purification from biogas or natural gas $\left(\mathrm{CO}_{2} / \mathrm{CH}_{4}\right)$ and $\mathrm{CO}_{2}$ capture in general $\left(\mathrm{CO}_{2} / \mathrm{N}_{2}\right)$.

Separation membranes can be made from ceramics, metals and polymers. The latter is of interest because they are less costly and less fragile but suffer from the famous permeability-selectivity trade-off known as the Robeson upper bounds [1]. For this reason different approaches are still under investigation to overcome this limitation and maximize the full potential of polymer membranes for gas separation. Unfortunately, there is no universal system that will work for any gas separation. For example, it has been reported that Ultem (polyetherimide) would performed best for $\mathrm{H}_{2} / \mathrm{N}_{2}, \mathrm{H}_{2} /$ $\mathrm{CH}_{4}$ and $\mathrm{O}_{2} / \mathrm{N}_{2}$ separation, while Matrimid (polyimide) would be better for $\mathrm{CO}_{2} / \mathrm{N}_{2}$ and $\mathrm{CO}_{2} / \mathrm{CH}_{4}$. Nevertheless, Ultrason (polyethersulfone) would be best for $\mathrm{O}_{2}$ enrichment. But these conclusions might be dependent on the operating conditions like pressure and temperature, as well as the inlet gas composition [2].
To improve on the polymer membrane efficiency, several methods/techniques have been proposed. Although a great deal of work has been done on synthesis (development of new polymers/copolymers), one simple way to control the permselectivity of available polymers is to modify the membrane structure. Some examples are presented next.

One of the most investigated structure is the production of mixed matrix membranes (MMM) [3]. This is done by adding functionalized nanoparticles into the polymer. This strategy opens the door to a wide range of particles (zeolites or metal organic frameworks), but several problems like optimum concentration, good dispersion, interfacial adhesion and pore blockage still need to be fully resolved.

Another way to improve the membrane performance is to use a multilayer structure. In this case, each layer has a specific task to control the chemical, mechanical and physical properties of the system depending on the gases and conditions. For example, a thin polydimethylsiloxane (PDMS) layer was used to control the permselectivity which was coated on a low-cost foamed polyethylene (PE) layer mainly acting as a mechanical support [4]. An easy way to modify the properties of polymer is via additives. These low molecular weight components can modify the matrix chemical, mechanical and physical properties, as well as the interactions between all the components including the diffusing gases. This was shown to be the case by adding aromatic carboxylic acids into Pebax (poly(ether-b-amide)) [5].

Membrane cost is still a limiting criterion for gas separation. To reduce the membranes cost, continuous processes must be used with commercial commodity polymers. For example, polyethylene 
was proposed to produce a foamed structure via extrusion [6]. With optimized processing conditions, it was possible to control the porous structure in terms of cell size, density and geometry, as well as the open-cell content to get a complete control of the resulting membrane permselectivities.

Although the membrane itself can be optimized, the module design and configuration must also be investigated in more details. For example, the type of membrane (flat, hollow fiber or spiral) must be selected [7]. Also, optimization of the complete separation process must be investigated to improve on the separation performance while taking into account the overall costs. This can be done using the concept of stage-cut [8].

Finally, the membrane production should be better controlled. For example, a clear relation between the rheological properties of the materials (multiphase and multicomponent solutions or melts) must be determined to get a better control on the final membrane structure (active layer thickness, porosity, orientation, crystallinity, etc.). Although some works are available on the shear rheology of these systems [9], their elongational behavior is still to be investigated.

\section{Conclusion}

Polymer membranes are very interesting for gas separation applications because they can be low cost, easy to process, flexible and low density. Since no single polymer can perform all the tasks required, this is why material selection and structural design must be done, not only on the membrane itself, but also on the modules used for industrial applications. Several solutions were proposed to improve on the membrane performances, but the optimum may be obtained only by a combination of several of them (synergistic effects). So, it is expected that important development will occur in the future and this can be done via experimental investigations to supply data for numerical models (validation).

\section{References}

1. Robeson LM (2008) The upper bound revisited. Journal of Membrane Science 320(1): 390-400.

2. Chen XY, Kaliaguine S, Rodrigue D (2017) A comparison between several commercial polymer hollow fiber membranes for gas separation. Journal of Membrane and Separation Technology 6(1): 1-15.

3. Paul DR, Kemp DR (1973) The diffusion time lag in polymer membranes containing adsorptive fillers. Journal of Polymer Science 93(1): 79-93.

4. Shahidi K, Rodrigue D (2018) Gas transport and mechanical properties of PDMS-TFS/LDPE nanocomposite membranes. Journal of Polymer Research 25(8): 179.

5. Meshkat S, Kaliaguine S, Rodrigue D (2019) Enhancing $\mathrm{CO}_{2}$ separation performance of Pebax ${ }^{\circledR}$ MH-1657 with aromatic carboxylic acids. Separation and Purification Technology 212(1): 901-912.

6. Razzaz Z, Mohebbi A, Rodrigue D (2018) Effect of processing conditions on the cellular morphology of polyethylene hollow fiber foams for membrane applications. Cellular Polymers 37(4-6): 169-188.

7. Chen XY, Kaliaguine S, Rodrigue D (2018) Correlation between performances of hollow fibers and flat membranes for gas separation. Separation \& Purification Reviews 47(1): 66-87.

8. Gilassi S, Taghavi SM, Rodrigue D, Kaliaguine S (2018) Simulation of gas separation using partial element stage cut modeling of hollow fiber membrane modules. AIChE Journal 64(5): 1766-1777.

9. Ullsperger J, Valek R (2018) Properties of polymer solutions intended for formation of hollow fibers membranes by inversion phases process. Applied Rheology 28(6): 65935.
Creative Commons Attribution 4.0

International License

For possible submissions Click Here
Submit Article

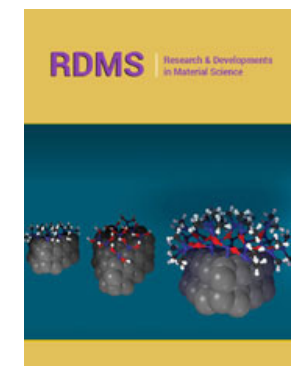

\section{Research \& Development in Material Science}

\section{Benefits of Publishing with us}

- High-level peer review and editorial services

- Freely accessible online immediately upon publication

- Authors retain the copyright to their work

- Licensing it under a Creative Commons license

- Visibility through different online platforms 